\title{
Management of severe complications following radical vaginal trachelectomy
}

\author{
Michele Mazzola $\cdot$ Vincenzo Miceli • Sarah Tardino • \\ Luigi Marsala $\cdot$ Roberto Tozzi
}

Received: 23 April 2010 /Accepted: 26 May 2010 /Published online: 16 June 2010

(C) Springer-Verlag 2010

\section{Introduction}

Uterine cervical cancer is the second most common cancer worldwide in females with 493,000 new cases per year and 273,000 deaths of which $80 \%$ is in developing countries; the incidence of uterine cervical cancer in developed countries is 14 per 100,000 per year with cancer mortality inferior to five per 100,000 per year [1].

Usually, $80 \%$ of uterine cervical neoplasms are squamous carcinomas and the majority of the remaining is adenocarcinomas [2]. In the developed countries, great improvements in screening techniques allows one to diagnose tumours at an earlier stage.

However, $38.6 \%$ of stage I carcinomas diagnosed in 2000 were women under the age of 40 (http://seer.cancer. gov/csr/1973_1999/overview/overview16.pdf, accessed 9 Nov 2009), with an obvious impact on fertility. Meantime, over the last two decades, there has been a delay of the maternity to the age 25-29 years, with an increasing number of women over 30 still childless [3]. Since 1902, the standard treatment for cervical cancer has been a radical hysterectomy. This surgery aims to remove the uterus, the parametrium and the upper third of the vagina, irremediably compromising the fertility. A significant

\footnotetext{
M. Mazzola $\cdot$ V. Miceli $\cdot$ S. Tardino $\cdot$ L. Marsala Department of Gynaecology and Obstetrics, San Raffaele Giglio Hospital, Contrada Pietrapollastra, Cefalù,

Palermo, Italy

R. Tozzi $(\bowtie)$

Department of Gynaecologic Oncology,

Oxford Cancer Centre,

Oxford, UK

e-mail: roberto.tozzi@obs-gyn.ox.ac.uk
}

number of patients with cervical cancer will be women in childbearing age, for which treatment will mean sterilisation. For the first time in 1987, Dargent performed a radical fertility sparing surgery for uterine cervical cancer, called radical vaginal trachelectomy (RVT) [4]. Since 1987, several studies have confirmed safe oncological results and reassuring obstetrical outcomes [5]. This case report describes the complications and its management that occurred to a nulliparous woman following a RVT, which was performed 5 years before.

\section{Case presentation}

In July 2009, a nulliparous 43-year-old woman presented to our attention complaining of pelvic pain and amenorrhea. In 2004, the patient was diagnosed with a FIGO stage IB1 squamous cell carcinoma of the cervix and underwent RVT with pelvic lymphadenectomy in another centre with a strong reputation for RVT. Since the operation, she never regained normal periods. Additionally, in the course of the following year, she was diagnosed with a bilateral uretericvaginal fistula. She underwent a left-side positioning of stent and a further operation to re-implant the right ureter. Subsequently, she recovered a normal life, with the exception of the persistent amenorrhea. She attended regular follow-up appointments with clinical examinations, smear and scans. No evidence of recurrence was ever detected. The patient presented to our attention a computed tomography (CT) scan performed elsewhere of the abdomen that showed bilateral multiloculated adnexal masses $(95 \times 67 \mathrm{~mm}$ right, $65 \times 43 \mathrm{~mm}$ left $)$, an enlarged uterine cavity with inhomogeneous contents, diffusely thickened pelvic fat with possible peritoneal nodulations and hydroureteronephrosis on the right and light ureteral 
hypotonicity on the left produced by the adnexal masses. To better investigate the clinical findings and the anatomy, a magnetic resonance imaging (MRI) of the pelvis was done. The MRI confirmed the presence of bilateral pelvic masses with contrast-enhancing content in the right one. Also, the endometrial cavity was found to be filled with a mixed liquid and solid content as a haematometra. A positron emission tomography scan was carried out to rule out a tumour recurrence. The findings were essentially negative for a tumour recurrence, although a nonspecific capitation was detected in the pelvis nearby the area of a possible sactosalpinx. The woman (weight $50 \mathrm{~kg}$, height $160 \mathrm{~cm}$ ) presented with normal abdomen, external genitalia and inferior third of the vagina. The isthmus of the uterus, which is usually sutured to the vagina following the RVT, was not successfully visualised at the examination with the speculum. This finding is not uncommon following the RVT. The hydronephrosis was responsible for a moderate rise of the creatinine, although the renal cortex was still well represented at the CT scan. A low haemoglobin level in the blood $(8.1 \mathrm{mg} / \mathrm{dl})$ was the only remarkable value. A provisional diagnosis of uterine stenosis with retrograde menstruation as the cause for the sactosalpinx and pelvic masses was made. The patient was offered the opportunity of surgery to rule out a tumour recurrence and mainly to take care of the adnexal masses responsible of the hydronephrosis. Finally, the aim was also to drain the haematometra that produced contraction and spasm of the uterus, hence the colicky pain. Initially, the patient underwent a cystoscopy with bilateral ureteral stenting. Then, during the same operation, at the examination under anaesthetics, it became clear that the vagina had flashed over the isthmus, rendering any probing impossible. Hence, a colpotomy was performed and an access to the uterine cavity was created, surrounded by thickly scarred fibrotic tissues. Under ultrasound guidance, the passage was dilated using Hegar's dilators. Finally, a copious discharge of haematic material was obtained, draining the haematometra. Additionally, a biopsy of the vagina was obtained and sent for histology. A 12 Ch Foley catheter was placed in the presumed uterine cavity with the goal to drain the haematometra and the sactosalpinx. The surgery was interrupted at this stage to confirm the absence of a tumour recurrence. The operation lasted about $80 \mathrm{~min}$. The histopathology report was negative for malignancy with only heavy fibrotic tissue. Based on a negative radiologic and histologic finding, the patient was considered diseasefree. After 6 days, the patient agreed on having a definitive treatment. Therefore, she underwent a laparoscopy. At the overview, a frozen pelvis was noticed with multiple tenacious adhesions and the right pelvic mass encased in the pelvic side wall. Following a careful and prolonged adhesiolysis, the uterus and tubes were visualised to be all matted together. Both tubes appeared dilated as in a sactosalpinx. The right tube was inseparable from the homolateral ovary. The right ureter that had previously been reimplanted was visualised and spared. A decision was made to remove the right ovary and the left tube. Then, the blood supply was discontinued with the right utero-ovarian and infundibolo-pelvic ligaments coagulated and cut. With further mobilisation on the right, the entire adnexal was resected with spillage of some dark blood as in endometriomas. The same procedure was performed on the left side, mobilising and extirpating the left salpinx, sparing the ipsilateral ovary. After checking for an accurate haemostasis, finally, the peritoneal cavity was copiously washed out and a Robinson drain was placed. This second operation lasted about 255 minutes. On the first postoperative day, the patient needed the transfusion of two units of blood because of a drop in the haemoglobin level to $5.7 \mathrm{mg} / \mathrm{dl}$, although no symptoms appeared. The patient was discharged in good general conditions on day 5 post-surgery. The histology confirmed the presence of bilateral endometriomas, but no tumour recurrence. The passage created at the time of surgery from the isthmus to the vagina closed again some few days later. Hence, it was agreed with the patient that she would go on taking an estroprogestinic pill to avoid a retrograde menstruation. At 8 months' follow-up, she is doing fairly well with very sporadic episodes of mild pelvic pain. The pelvic masses have not recurred and the hydronephrosis is completely regressed.

\section{Conclusion}

Radical vaginal trachelectomy with pelvic lymphadenectomy has two targets: sparing the fertility and treating the tumour. Considering the increase of early diagnosis and the age of diagnosis, it seems that a significant number of women younger than 40 and with early-stage cervical cancer will be eligible for RVT [6]. This is also encouraged by the excellent oncological outcomes: 5 years of disease-free survival in $90.8 \%$ of cases, not any different than the outcome of radical surgery [7]. In addition, RVT seems to carry a low morbidity [5]. Finally, pregnancy seems a feasible outcome and, despite that $57 \%$ of the patients who undergone RVT did not try to get pregnant $70 \%$ of the remaining succeeded to conceive once or more than once [8]. Nonetheless, RVT remains a major surgery. Despite being associated with a low morbidity, the complications can be quite severe. One of them, the cervical stenosis, is poorly described in the medical literature $[9,10]$. Also, when compared to radical hysterectomy, RVT has been reported to carry less short-term morbidity (blood loss, transfusions rate, febrile conditions, hospital stay, regular analgesia, bladder 
hypotonicity) and less operative complications (vascular injuries, cystotomy) but more specific side effects (dysmenorrhoea, dysplastic smears, irregular bleedings, discomfort from the cervical cerclage, excessive vaginal discharge, isthmic stenosis, amenorrhea) and more long-term complications (vaginal granulation tissue, chronic pelvic pain, dyspareunia, neuropathy, vaginal discharge, psychological problems) [9]. So, despite considering RVT as a safe surgery with promising fertility outcome, it may provoke serious complications even in the most experienced hands. It should be performed in specialised centres and the complexity of the surgery should be clearly explained to the patient.

Conflicts of interest The authors report no conflicts of interest. The authors alone are responsible for the content and writing of the paper.

\section{References}

1. Sankaranarayanan R, Ferlay J (2006) Worldwide burden of gynaecological cancer: the size of the problem. Best Pract Res Clin Obstet Gynaecol 20(2):207-225

2. Berrington de González A, Green J (2007) Comparison of risk factors for invasive squamous cell carcinoma and adenocarcinoma of the cervix: collaborative reanalysis of individual data on 8,097 women with squamous cell carcinoma and 1,374 women with adenocarcinoma from 12 epidemiological studies. Int J Cancer 120(11):2525

3. Hamilton BE, Ventura SJ (2006) Fertility and abortion rates in the United States, 1960-2002. Int J Androl 29(1):34-45

4. Dursun P, LeBlanc E, Nogueira MC (2007) Radical vaginal trachelectomy (Dargent's operation): a critical review of the literature. Eur J Surg Oncol 33(8):933-941

5. Plante M, Renaud MC, François H, Roy M (2004) Vaginal radical trachelectomy: an oncologically safe fertility-preserving surgery. An updated series of 72 cases and review of the literature. Gynecol Oncol 94(3):614-623

6. Covens A, Rosen B, Murphy J, Laframboise S, DePetrillo A, Lickrish G, Colgan T, Chapman W, Shaw P (2001) Changes in the demographics and perioperative care of stage IA2/IB1 cervical cancer over the past 16 years. Gynecol Oncol 81:133137

7. Hertel H, Köhler C, Grund D, Hillemanns P, Possover M, Michels W, Schneider A, German Association of Gynecologic Oncologists (AGO) (2006) Radical vaginal trachelectomy (RVT) combined with laparoscopic pelvic lymphadenectomy: prospective multicenter study of 100 patients with early cervical cancer. Gynecol Oncol 103(2):506-511

8. Boss EA, van Golde RJ, Beerendonk CC, Massuger LF (2005) Pregnancy after radical trachelectomy: a real option? Gynecol Oncol 99(3 Suppl 1):S152-S156

9. Alexander-Sefre F, Chee N, Spencer C, Menon U, Shepherd JH (2006) Surgical morbidity associated with radical trachelectomy and radical hysterectomy. Gynecol Oncol 101(3):450-454

10. Martin A, Torrent A (2010) Laparoscopic nerve-sparing radical trachelectomy: surgical technique and outcome. J Minim Invasive Gynecol 17(1):37-41 